\title{
Some New Directions for Publication in the Journal of Psychopathology and Behavioral Assessment: New Constructs, Physiological Assessment, Worldwide Contribution, and Economics
}

\author{
Randall T. Salekin
}

Published online: 16 February 2014

(C) Springer Science+Business Media New York 2014

\begin{abstract}
This issue marks the beginning of my sixth year as Editor-in-Chief of Journal of Psychopathology and Behavioral Assessment (JPBA). Due to excellent submissions, the journal has had impressive success in the past 5 years with the impact factor doubling in that time. Our team remains committed to soliciting and publishing the very best articles in order to continue this trend and to place the most informative science in the pages of $J P B A$. With high-quality submissions, the journal has been able to present chief developments in the science and practice of assessment and psychopathology. Scholarly manuscripts have also included important papers on intervention. All these studies have provided rich journal content and the editorial team for the journal is committed to improving manuscript quality through constructive feedback. In next few years, I would like to encourage authors to write articles that better inform ways to assess, understand, and treat psychopathology. New directions that may aid in this upcoming research adventure include addressing $\mathrm{RDoC}$ constructs, utilizing multi-time point investigations, utilizing physiological assessment, collaborating in worldwide contributions, and considering the economics of assessment and intervention. I am excited about the up-coming year of editing the journal and invite authors to take advantage of the range of opportunities for publication in this journal.
\end{abstract}

\section{Introduction}

The primary focus of our journal is on the publication of scholarly articles on the assessment of psychologically

R. T. Salekin $(\bowtie)$

Department of Psychology, University of Alabama, Tuscaloosa, AL, USA

e-mail: rsalekin@ua.edu relevant concepts. The journal is also interested in treatment articles particularly if the manuscripts provide a better understanding of psychopathology. The field is rapidly changing and as the field changes the types of articles that most frequently make their way into the pages of the journal have something new and innovative to offer. An article that is accepted into the Journal of Psychopathology and Behavioral Assessment generally reflects mastery of the assessment, psychopathology, and intervention area that the author is studying. In my first and second editorials for the journal (Salekin 2009, 2011), I highlighted several points that I would like to reiterate and expand upon here. I also feel compelled to state what types of articles are less compelling at this point in the journal's life. This advice is intended to help authors shape their articles to have a more meaningful impact on the field and to be of importance to the reader of the journal.

There are six main areas that would strengthen submissions to the journal including 1) new concepts, 2) longitudinal research, 3) measuring the benefits of psychotherapy 4) physiological assessment, 5) worldwide research, and 6) the economics of assessment and intervention. I cover each of these topics before briefly discussing the review process.

New Domains of Study—-The Research Domain Criteria (RDoC)

The National Institute of Mental Health recently launched their Research Domain Criteria (RDoC) onto the mental health scene. The $\mathrm{RDoC}$ is an initiative designed to promote research on the brain mechanism underlying mental health concerns. The RDoC describes five functional domains including Negative Affect, Positive Affect, Cognition, Social Processes, and Regulatory systems (Insel et al. 2010; Sanislow et al. 2010). Part of the reason for this initiative is to synthesize research domains and to facilitate the 
development of interventions that target these brain mechanisms. Thus, a chief focus of the RDoC includes opening up new lines of research examining patients' responses on biological measures. Because of the importance of this newly developing area, I would encourage researchers to submit manuscripts to JPBA that consider RDoC concepts and physiological measurement. I will cover the topic of physiological assessment in more detail below. There is also a need for integration of the various systems. Thus, studies that shed light on the interconnections between the RDoC and DSM-5 and ICD-11 constructs are encouraged.

\section{Examining the Process with Longitudinal Research}

In my initial editorials (Salekin 2009, 2011), I stressed the importance of single time point research for establishing nomological networks for disorders and conditions. I continue to see this as an important area of study. However, if studies are single time point studies they should be on constructs from new nosological systems such as those delineated in the DSM5, the ICD-11, or the RDoC. I would also continue to challenge researchers to examine disorders and conditions across time. This remains an important agenda item where investigators could note both risk and protective factors that play an important role in bringing about, or protecting against, the development of disorders. Investigations of the mediators or potential causes of disorders can be key to better understanding mental health conditions. At this point in the journals' development, simple replications of factor structures, while important for the measures themselves, are less desirable for the journal. This is because these types of replications might be the better suited for presentation in the test manuals themselves. Factor structure papers with a conceptual component, such as the examination of extra test criteria, including convergent discriminant validity analyses, could be considered for publication.

\section{Measuring the Benefits of Psychotherapy}

There is a need for more research on the measurement of outcome in psychotherapy. Recently, we highlighted several areas where research could further inform assessment and thus better understand change across interventions. Specifically, along with the need to integrate systems (DSM-5, ICD-10, $\mathrm{RDoC}$ ), and examine psychological concepts longitudinally, there is also a need for test development and measurement in the context of idiographic and nomothetic treatment designs (Salekin et al. 2013). There also continues to be a need for change sensitive measures in these designs. Studies which also show the integration between idiographic and nomothetic designs could further assist with knowledge in this area. And, as previously mentioned, tapping novel areas of measurement (e.g., biological functioning, contextual factors) using emerging technologies would be productive. We are in a good position to build on the work that was ignited by Smith and Glass (1977) to quantitatively measure the benefits of psychological therapy.

\section{Physiological Assessment}

Physiological assessment including EEG and new imaging technologies have had a demonstrable impact on the landscape of scientific research. Developments in neuroimaging during the past quarter century have increasingly made it possible to investigate the differential involvement of particular brain regions in normal and disordered thought in humans. Prior to these developments, the neurophysiological structures and functions associated with psychological states and processes where limited to animal research and models, postmortem examinations, EEG measurements, and observations of the occasional situation where an unfortunate individuals suffered trauma to or disorders of the brain (Caccioppo et al. 2008). The highly detailed three dimensional images provided by neuroimaging, modeling statistical properties of the working brain as well as EEG measurement may well add to our understanding of psychopathology, assessment and intervention. When possible such technology should be considered for inclusion in psychopathology and intervention research studies (Salekin et al. 2012). In addition, although not covered in detail here, the journal is very interested in studies on molecular and behavior genetics. There is considerable room for growth and additional knowledge on these topics.

\section{Worldwide Research}

The journal is keenly interested in research on psychopathology, assessment, and intervention science worldwide. Nationality, culture, and ethnicity can be important variables to study, and rich moderators in need of theory driven science to inform our understanding of psychopathology across the globe. Accelerated development of science and dissemination of this research can only help fuel a better understanding of the mental health problems that are experienced across the planet. Collaborative efforts will improve science and will help speed the recognition of problems that affect citizens, such as poverty, low levels of nutrition, natural disasters, war, and interpersonal violence. Research on psychopathology and assessment are needed from a global perspective. Moreover, economical ways of disseminating knowledge regarding what we know about psychopathology and how to treat it are needed. This topic is covered briefly below.

\section{Economics of Intervention}

The need to consider new ways to assess and intervene with the population is needed. This may include writing to the broader community. This may come in the form of self-help 
books geared to the general public, or manuals designed for parents with troubled youth, to see if the parents themselves can start to alleviate maladaptive conditions (e.g., oppositional defiant disorder). We also need to find ways to see if such advice received, is effectively put to use. Although this is just one possible suggestion, there are likely other ways to examine the economics of assessment and treatment of psychopathology across the world (Kazdin and Rabbitt 2013). Such investigations and research reports are encouraged.

\section{Review Process}

Our team is looking forward to reading and reviewing research reports and providing knowledgeable and scholarly reviews of each manuscript. The Editorial Board has increased in size and with this increase the board has a wide range of expertise. The journal has also acquired an excellent Associate Editor Team with Andres De Los Reyes, Daniel Gros, and Anne-Marie Iselin joining Jennifer Tackett and myself in the handling of manuscripts. Should a manuscript require additional expertise not reflected on the board, we will seek additional reviewers with requisite knowledge. As always, whether or not an article is accepted into the journal, we will strive to give authors constructive feedback. Article reviews can be critical at times with comments that seem more like personal attacks than constructive feedback (Sternberg, 2003). We will continue to discourage this practice. The editorial team has been successful at avoiding these types of reviews. Finally, the journal aims for speedy feedback and that will be maintained as a goal. As we have received more submissions, this became a challenge, but the journal has increased the number of AEs editing for the journal. We strive for professionalism and our team recognizes the importance of time efficiency and we will also work to move articles to print shortly after they have been accepted.

\section{Closing Comments}

The journal is flourishing and we hope to continue this upward trend. As always, we hope to accept articles from multiple perspectives (biological, cognitive, psychodynamic, behavioral, humanistic, positive psychology, prospective psychology, and so on) and from a variety of sources (e.g., interview, self- report observation) especially those including physiological measurement (e.g., brain imaging, EEG, etc.). I will continue to consider articles on a wide array of psychopathology as well as articles that focus on human well-being. I welcome suggestions for articles, special sections, and programs of research. The journal's diverse content allows for a unique combination of articles ranging from assessment to psychopathology to intervention. The links between the big three might help us better understand the etiology and treatment of disorders. Authors are assured that as the impact of the journal continues to rise a rather large audience will receive their articles directly. On that final note, I would like to thank all the authors and reviewers that have contributed to the journal thus far and to encourage researchers to continue to submit their impressive scientific papers to JPBA!

\section{References}

Caccioppo, J. T., Berntson, G. G., \& Nusbaum, H. C. (2008). Neuroimaging as a new tool in the toolbox of psychological science. Current Directions in Psychological Science, 17, 62-67.

Insel, T., Cuthbert, B., Garvey, M., Heinssen, R., Pine, D. S., Quinn, K., \& Wang, P. (2010). Research domain criteria (RDoC): Toward a new classification framework for research on mental disorders. The American Journal of Psychiatry, 167, 748-757.

Kazdin, A. E., \& Rabbitt, S. M. (2013). Novel models for delivering mental health services and reducing the burdens of mental illness. Clinical Psychological Science, 1, 170-191.

Salekin, R. T. (2009). Psychopathology and assessment: Contributing knowledge to science and practice. Journal of Psychopathology and Behavioral Assessment, 31, 1-6.

Salekin, R. T. (2011). Publication opportunity in the Journal of Psychopathology and Behavioral Assessment. Journal of Psychopathology and Behavioral Assessment, 33, 1-2.

Salekin, R. T., Tippey, J., \& Allen, A. (2012). Treatment of conduct problem youth with interpersonal callous traits using mental models: Measurement of risk and change. Behavioral Sciences and the Law, $30,470-486$

Salekin, R. T., Jarrett, M. A., \& Adams, E. W. (2013). Assessment and measurement of change considerations in psychotherapy research. In J. Comer \& P. K. Kendall (Eds.), The Oxford handbook of research strategies for clinical psychology (pp. 103-119). New York: Oxford University Press.

Sanislow, C. A., Pine, D. S., Quinn, K. J., Kozak, M. J., Garvey, M. A., Heinssen, R. K., \& Cuthbert, B. N. (2010). Developing constructs for psychopathology research: Research Domain Criteria. Journal of Abnormal Psychology, 119, 631-639. 